\title{
磷酸酯催化硫醚及缩硫醛氧化成亚砜反应
}

\author{
张俊琦 ${ }^{a}$ 李梅秋 ${ }^{a}$ 王兴旺*, $a$ 张 勇 $*, b \quad$ 贾 俊 $*, b$ \\ ( ${ }^{a}$ 苏州大学材料与化学化工学部 苏州 215123) \\ ( ${ }^{b}$ 苏州大学分析测试中心 苏州 215123)
}

\begin{abstract}
摘要 在室温条件下, 以过氧化氢水溶液为氧源, 非手性磷酸作为催化剂催化硫醚与缩硫醛的氧化反应, 以良好到优 秀的收率合成一系列亚砜化合物. 该方法反应条件温和, 时间短, 收率高, 且非对映选择性高.

关键词 硫醚; 缩硫醛; 氧化; 亚砜; 磷酸酯

\section{Oxidation of Thioether and Mercaptal to Sulfoxide Catalyzed by Hydrogen Phosphates}

\author{
Zhang, Junqi $^{a} \quad$ Li, Meiqiu $^{a} \quad$ Wang, Xingwang*,a ${ }^{*}$ Zhang, Yong ${ }^{*, b} \quad$ Jia, Jun ${ }^{*, b}$ \\ ( ${ }^{a}$ College of Chemistry, Chemical Engineering and Materials Science, Soochow University, Suzhou 215123) \\ ( ${ }^{b}$ Analysis and Testing Center, Soochow University, Suzhou 215123)
}

\begin{abstract}
Hydrogen phosphates have been explored as organocatalysts for the oxidation of a series of aryl alkyl sulfides and 1,3-dithianes derived from aldehydes with aqueous hydrogen peroxide $\left(\mathrm{H}_{2} \mathrm{O}_{2}\right)$ as the terminal oxidant. The corresponding sulfoxides are obtained in moderate to excellent yield (up to 99\%) with excellent diastereoselectivity (up to $>99: 1 d r$ ).

Keywords thioether; mercaptal; oxidation; sulfoxide; phosphates
\end{abstract}

亚砜类化合物 ${ }^{[1]}$ 具有重要的生物活性, 药物化学家 通过对亚砜类化合物的结构进行修饰，可以提高其药效 活性 ${ }^{[2]}$, 如抗溃疡、抗病毒、抗 HIV-1、抗肿瘤等. 在农 药领域，亚砜类化合物作为杀菌剂和除草剂具有用量少 低污染的优点. 亚砜类化合物中因含有半极性基团而成 为是一种软碱类中性萃取剂, 对软酸类的贵金属有特殊 亲和力，故其对贵金属萃取率高，选择性好等.

鉴于亚砜是许多天然产物及药物中间体的中重要 组成部分, 因此, 发展高效快捷合成亚砜的方法具有重 要的意义, 也是一个较为热点的研究领域. 除了过渡金 属催化的硫醚氧化外 ${ }^{[3]}$, 有机小分子催化的的方法主要 有以下几种: 硫脲为催化剂, 过氧叔丁醇为氧源合成亚

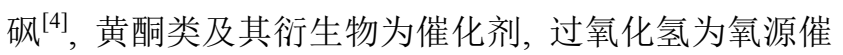
化合成亚砜 ${ }^{[5 \sim 8]}$, 及其他一些有机分子催化的合成方 法 ${ }^{[9 \sim 17]}$. 在这些研究方法中, 黄酮类及其衍生物为催化 剂催化合成亚砜的方法被广泛应用. 最近, Wang 和 List 课题组 ${ }^{[18]}$ 以手性的 Brønsted 酸作为催化剂催化不对称
的硫醚氧化反应，获得良好到优秀的手性诱导. 在这里, 我们以过氧化氢水溶液为氧源, 以磷酸二苯酯为催化 剂, 催化硫醚及缩硫醛的氧化, 能高产率得到亚砜类化 合物，砜作为反应的副产物基本上检测不到. 该催化剂 体系反应时间短，反应后处理简便，目标产物收率高.

\section{1 结果与讨论}

本实验以磷酸二苯酯为催化剂, 首先, 以苯基甲基 硫醚(1a) 氧化成亚砜反应为模板, 氯仿为溶剂, 以 $\mathrm{H}_{2} \mathrm{O}_{2}$ $(50 \%)$ 为氧源, 对反应催化剂进行篎选, 反应结果如表 1 所示. 用商业可得的亚磷酸二甲酯(I)来催化该反应, 在 室温条件下，三氯甲烷为溶剂反应 $12 \mathrm{~h}$, 以 $30 \%$ 的收率 得到目标化合物 $\mathbf{2 a}$ (表 1, Entry 1); 接下来尝试增加催化 剂的位阻来提高产物的收率, 当以亚磷酸二乙酯(II)为 催化剂时, 产物收率下降至只有 $20 \%$ (表 1, Entry 2). 而 以亚磷酸二异丙酯(III)催化该反应时, 可以以 $55 \%$ 的收 率得到目标化合物 $\mathbf{2 a}$ (表 1, Entry 3). 可是当用位阻更大

\footnotetext{
*E-mail: wangxw@suda.edu.cn; zhang-qinghe@263.net; jiajun@suda.edu.cn Received May 6, 2015; revised June 7, 2015; published online July 8, 2015. Project supported by the National Natural Science Foundation of China (No. 21272166). 国家自然科学基金(No. 21272166)资助项目.
} 
的亚磷酸二苯酯 $(\mathrm{IV})$ 催化该反应时, 产物的收率 $60 \%$ (表 1, Entry 4). 我们继续寻找合适的催化剂进行尝 试, 令我们高兴的是磷酸二苯酯 $(\mathbf{V})$ 催化该反应时, 能 以 $80 \%$ 的收率得到亚砜化合物 $\mathbf{2 a}$ (表 1 , Entry 5), 而磷酸 酯类催化剂 $\mathbf{V I} \sim$ VIII 催化该反应分别以 $70 \%$ 、15\%、 $20 \%$ 的收率得到目标产物 $2 \mathbf{a}$ (表 1, Entries 6 8). 因此, 磷酸二苯酯 $(\mathbf{V})$ 为催化该反应的最佳催化剂, 接下来我 们将反应的浓度从 $0.25 \mathrm{~mol} / \mathrm{L}$ 提高到 $0.5 \mathrm{~mol} / \mathrm{L}$ 时, 发现 产物的收率明显提高到 90\%(表 1, Entry 9).

表 1 反应催化剂的篮选 ${ }^{a}$

Table 1 Screening different catalysts
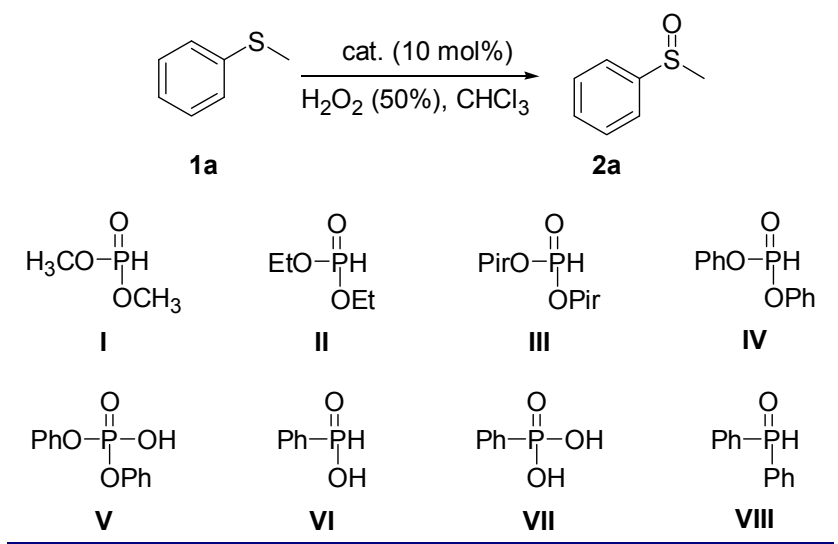

\begin{tabular}{clcc}
\hline Entry & Cat. & Time/h & Yield $^{b} \%$ \\
\hline 1 & I & 12 & 30 \\
2 & II & 12 & 20 \\
3 & III & 12 & 55 \\
4 & IV & 12 & 60 \\
5 & V & 12 & 80 \\
6 & VI & 12 & 70 \\
7 & VII & 12 & 15 \\
8 & VIII & 12 & 20 \\
9 & V & 10 & $90^{\mathrm{c}}$ \\
\hline
\end{tabular}

${ }^{a}$ Reactions were performed with 1a $(0.5 \mathrm{mmol}), \mathrm{H}_{2} \mathrm{O}_{2}\left(50 \%\right.$ in $\mathrm{H}_{2} \mathrm{O}, 1.2$ equiv.), and catalyst $\mathbf{I} \sim$ VIII $(10 \mathrm{~mol} \%)$ in $\mathrm{CHCl}_{3}(2.0 \mathrm{~mL}) .{ }^{b}$ Isolated yield. ${ }^{c}$ In $1.0 \mathrm{~mL} \mathrm{CHCl}_{3}$.

接下来以磷酸二苯酯 $\mathbf{V}(10 \mathrm{~mol} \%)$ 为催化剂, 反应 的浓度 $0.5 \mathrm{~mol} / \mathrm{L}$, 对反应的溶剂及过氧化氢水溶液的 量等反应条件进行优化, 反应结果如表 2 所示. 从表 2 可以看出, 四氢呋喃、1,4-二氧六环、乙醚为反应介质 时, 能分以 $55 \% 、 70 \% 、 50 \%$ 的收率得到产物(表 2, Entries $6,7,11)$; 反应以质子性溶剂乙醇、水作为溶剂时, 只能 以 $30 \%$ 和 $25 \%$ 的收率得到产物(表 2, Entries 2, 4); 而非 极性非质子性溶剂甲苯为溶剂时, 目标化合物 $\mathbf{2 a}$ 的收 率也只有 $65 \%$ (表 2, Entry 5); 我们发现极性非质子性溶 剂二氯甲烷、乙腈、乙酸乙酯、丙酮及三氯甲烷为反应 介质时，除了丙酮仅以 $32 \%$ 收率得到产物 $2 \mathbf{a}$ 外(表 2, Entry 9), 其他溶剂能以 $80 \% \sim 90 \%$ 的收率得到目标产 物(表 2 , Entries 1, 3, 8, 12). 进而以 1,2-二氯乙烷为反应
溶剂时, 以 95\%的收率得到产物 2a(表 1, Entry 10). 因 此, 反应以 1,2 -二氯乙烷作为反应介质时更有利于反应 的进行. 为了提高反应效率我们尝试增加过氧化氢水溶 液的用量, 我们发现过氧化氢水溶液的量从 1.2 equiv. 增加到 1.5、2.0 equiv. 时, 反应效率明显加快, 反应收率 分别提高至 93\%、99\%(表 2, Entries 13, 14), 且没有观察 到副产物. 因此, 当过氧化氢水溶液的量是 $\mathbf{1 a}$ 的 2.0 equiv.时，对反应最有利(表 2, Entry 14).

表 2 反应溶剂的笁选 ${ }^{a}$

Table 2 Screening different solvents

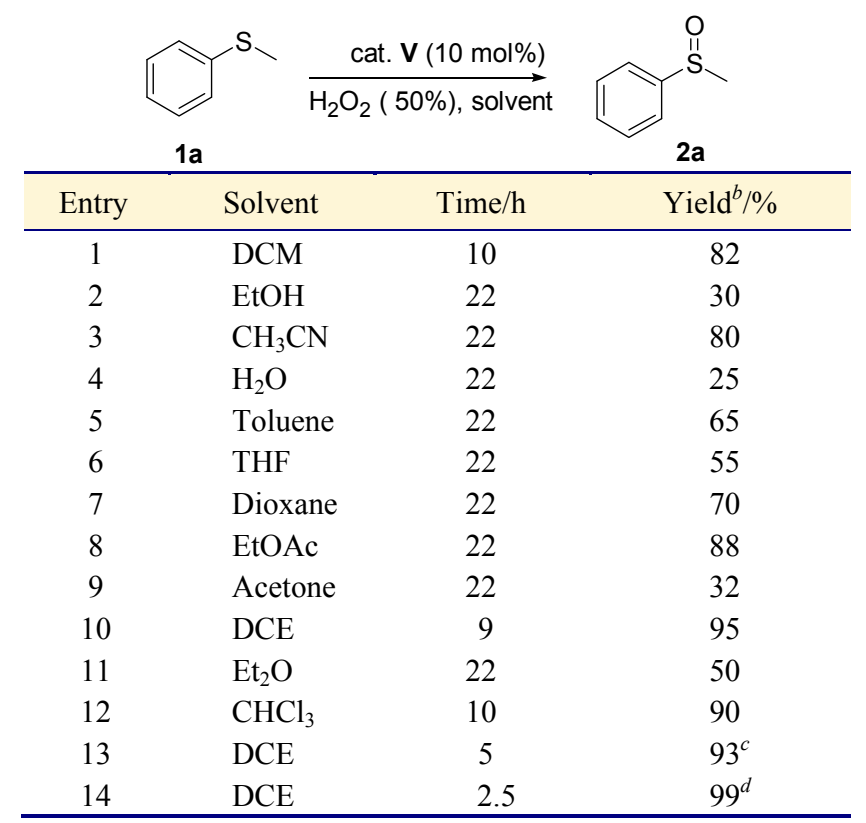

${ }^{a}$ Reactions were performed with 1a $(0.5 \mathrm{mmol}), \mathrm{H}_{2} \mathrm{O}_{2}\left(50 \%\right.$ in $\mathrm{H}_{2} \mathrm{O}, 1.2$ equiv.), and catalyst $\mathbf{V}(10 \mathrm{~mol} \%)$ in solvent $(1.0 \mathrm{~mL}) .{ }^{b}$ Isolated yield. ${ }^{c} \mathrm{H}_{2} \mathrm{O}_{2}$ (50\% in $\mathrm{H}_{2} \mathrm{O}, 1.5$ equiv.). ${ }^{d} \mathrm{H}_{2} \mathrm{O}_{2}\left(50 \%\right.$ in $\mathrm{H}_{2} \mathrm{O}, 2.0$ equiv.).

我们以磷酸二苯酯 $(\mathbf{V})(10 \mathrm{~mol} \%)$ 为催化剂, 反应的 浓度 $0.5 \mathrm{~mol} / \mathrm{L}, 1,2$-二氯乙烷作为反应介质, 氧化剂 $\mathrm{H}_{2} \mathrm{O}_{2}$ 的用量为 2.0 equiv., 对反应底物的普适性进行考 察，反应结果如表 3 所示. 苯基甲基硫醚 $2.5 \mathrm{~h}$ ，以 $99 \%$ 的收率得到目标化合物(表 3, Entry 1). 对甲氧基、对甲 基苯基甲基硫醚 $(\mathbf{1 b}, \mathbf{1 g})$ 作为反应底物分别反应 1.5、3.0 $\mathrm{h}$, 能以 $96 \%$ 、90\%的收率得到目标化合物 $2 \mathbf{b}$ 和 $\mathbf{2 g}$ (表 3 , Entries 2, 7). 苯基苠基硫醚(1c)、苯基乙基硫醚(1h) 作为反应底物，分别反应 $3.5 、 4.0 \mathrm{~h}$ 后，分别以 $94 \%$ 、 90\%的收率得到目标化合物 $\mathbf{2 c}$ 和 $\mathbf{2 h}$ (表 3, Entries 3, 8). 对溴、对氯苯基甲基硫醚 $1 \mathrm{e}$ 和 $\mathbf{1 f}$, 分别反应 $2.5 、 3.0 \mathrm{~h}$, 能以 $93 \%$ 、92\%的收率得到目标化合物(表 3, Entries 4, 5). 邻溴苯基甲基硫醚 $2.0 \mathrm{~h}$, 能以 $99 \%$ 的收率得到目标 化合物(表 3, Entry 6).

我们在以上优化好的反应条件来考察 1,3-缩硫醛 $3 \mathbf{a}$ 的氧化时, 发现 1,3-缩二硫醛产物 $4 \mathbf{a}$ 在 1,2-二氯乙烷 中的溶解性不好, 不利于反应的进行, 而且以 2.0 equiv. 
表 3 反应底物的拓展 ${ }^{a}$

Table 3 Substrates scope in the reaction

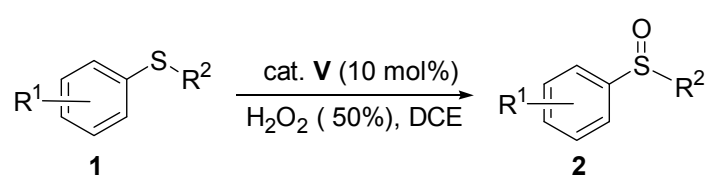

\begin{tabular}{clccc}
\hline Entry & \multicolumn{1}{c}{$\mathrm{R}^{1}, \mathrm{R}^{2}$} & Product & Time $/ \mathrm{h}$ & Yield $/ \%$ \\
\hline 1 & $\mathrm{R}^{1}=\mathrm{H}, \mathrm{R}^{2}=\mathrm{CH}_{3}(\mathbf{1 a})$ & $\mathbf{2 a}$ & 2.5 & 99 \\
2 & $\mathrm{R}^{1}=p-\mathrm{OCH}_{3}, \mathrm{R}^{2}=\mathrm{CH}_{3}(\mathbf{1 b})$ & $\mathbf{2 b}$ & 1.5 & 96 \\
3 & $\mathrm{R}^{1}=\mathrm{H}, \mathrm{R}^{2}=\mathrm{Bn}(\mathbf{1 c})$ & $\mathbf{2 c}$ & 3.5 & 94 \\
4 & $\mathrm{R}^{1}=p-\mathrm{Br}, \mathrm{R}^{2}=\mathrm{CH}_{3}(\mathbf{1 d})$ & $\mathbf{2 d}$ & 2.5 & 93 \\
5 & $\mathrm{R}^{1}=p-\mathrm{Cl}, \mathrm{R}^{2}=\mathrm{CH}_{3}(\mathbf{1 e})$ & $\mathbf{2 e}$ & 3.0 & 92 \\
6 & $\mathrm{R}^{1}=o-\mathrm{Br}^{2} \mathrm{R}^{2}=\mathrm{CH}_{3}(\mathbf{1 f})$ & $\mathbf{2 f}$ & 2.0 & 99 \\
7 & $\mathrm{R}^{1}=p-\mathrm{CH}_{3}, \mathrm{R}^{2}=\mathrm{CH}_{3}(\mathbf{1 g})$ & $\mathbf{2 g}$ & 3.0 & 90 \\
8 & $\mathrm{R}^{1}=\mathrm{H}, \mathrm{R}^{2}=\mathrm{C}_{2} \mathrm{H}_{5}(\mathbf{1 h})$ & $\mathbf{2 h}$ & 4.0 & 90 \\
\hline
\end{tabular}

${ }^{a}$ Reactions were performed with $\mathbf{1 a} \sim \mathbf{1 h}(0.5 \mathrm{mmol}), \mathrm{H}_{2} \mathrm{O}_{2}\left(50 \%\right.$ in $\mathrm{H}_{2} \mathrm{O}, 1.2$ equiv.), and catalyst $\mathbf{V}(10 \mathrm{~mol} \%)$ in DCE $(1.0 \mathrm{~mL}) .{ }^{b}$ Isolated yield.

的过氧化氢水溶液为氧化剂, 反应体系较为复杂. 当把 过氧化氢水溶液的量降到 1.2 equiv. 时, 反应 $1 \mathrm{~h}$, 能以 99: 1 的非对映选择性得到目标产物 4a. 因此为了提高 反应的收率我们对反应溶液进行篎选, 催化结果如表 4 所示. 反应在不同的反应介质中都能快速反应，在 1,2二氯乙烷和二氯甲烷中反应产物 $\mathbf{4 a}$ 的收率只有 $78 \%$ 、 $76 \%$ (表 4, Entries 5, 7), 而以乙酸乙酯作为反应的介质 时, 能以 $90 \%$ 的收率得到 $\mathbf{4 a}$, 反应非均相本质表明产物 在乙酸乙酯中溶解度较差(表 4, Entry 1). 我们用甲醇、 四氢呋喃、乙腈作反应的溶剂时，能以 $85 \% \sim 89 \%$ 的收 率得到目标化合物 $4 \mathrm{a}$ (表 4, Entries 2, 3,6), 反应在氯仿 中能以 $95 \%$ 的收率获得产物 $\mathbf{4 a}$ (表 4, Entry 4). 因此我们 选择氯仿作溶剂, 对底物进行拓展.

表 4 反应溶剂的篮选 ${ }^{a}$

Table 4 Screening different solvents

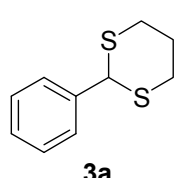
$\frac{\text { cat. V }(10 \text { mol\%) }}{\stackrel{\mathrm{H}_{2} \mathrm{O}_{2}(50 \%) \text {, solvent }}{(.0 \mathrm{~h}}}$

\begin{tabular}{clc}
\hline 3a & \multicolumn{1}{c}{ 4a } \\
\hline Entry & \multicolumn{1}{c}{ Solvent } & Yield $^{b} \%$ \\
\hline 1 & EtOAc & 90 \\
2 & $\mathrm{CH}_{3} \mathrm{OH}$ & 88 \\
3 & $\mathrm{THF}$ & 85 \\
4 & $\mathrm{CHCl}_{3}$ & 95 \\
5 & $\mathrm{DCE}$ & 78 \\
6 & $\mathrm{CH}_{3} \mathrm{CN}$ & 89 \\
7 & $\mathrm{DCM}$ & 78 \\
\hline
\end{tabular}

${ }^{a}$ Reactions were performed with 3a $(0.5 \mathrm{mmol}), \mathrm{H}_{2} \mathrm{O}_{2}\left(50 \%\right.$ in $\mathrm{H}_{2} \mathrm{O}, 1.2$ equiv.) and catalyst $\mathrm{V}(10 \mathrm{~mol} \%)$ in solvent $(1.0 \mathrm{~mL}) .{ }^{b}$ Isolated yield.

以磷酸二苯酯 $\mathbf{V}(10 \mathrm{~mol} \%)$ 为催化剂, 氯仿为溶剂, 氧化剂 $\mathrm{H}_{2} \mathrm{O}_{2}$ 的用量为 1.2 equiv., 对一系列 1,3-缩二硫
芳醛衍生物进行考察(表 5). 首先考察芳环取代基效应 对反应产物的收率及非对映选择性的影响。当底物 $3 \mathbf{b} \sim 3 \mathbf{g}$ 的苯环对位带有吸电子基团 $\left(\mathrm{F} 、 \mathrm{Cl} 、 \mathrm{Br} 、 \mathrm{CF}_{3}\right.$ 、 $\mathrm{CN} 、 \mathrm{NO}_{2}$ ) 时, 反应能在 $1.5 \sim 2.5 \mathrm{~h}$ 内完成, 能以 $86 \% \sim$ 97\%的收率以及大于 $99 ： 1$ 的非对映选择性得到目标化 合物 $\mathbf{4 b \sim 4} \sim \mathbf{g}$ (表 5, Entries 2 7); 当芳环的对位带有供 电子基团 $\left(\mathrm{CH}_{3} 、 i-\mathrm{Pr}\right)$ 的 $\mathbf{3 h}$ 和 $3 \mathbf{i}$ 作为反应底物时, 能分 别以 $93 \%$ 和 96\%的收率及大于 $99 ： 1$ 非对映选择性得到 产物 $4 \mathbf{h}$ 和 $4 \mathbf{i}$ (表 5, Entries 8,9); 当芳环的邻位带有供电 子基团 $\mathrm{OCH}_{3}$ 时, 以 $94 \%$ 的收率得到反应产物 $4 \mathbf{j}$, 但是 产物 $4 \mathbf{j}$ 的非对映选择性下降至 $94 ： 6$ (表 5, Entry 10). 当 芳环的邻位带有吸电子基团 $(\mathrm{F} 、 \mathrm{Cl})$ 时，反应产物 $4 \mathbf{k} 、 4 \mathbf{l}$ 的收率 $(81 \%$ 和 $82 \%)$ 非对映选择性为 $(92: 8$ 和 $89: 11)$ 都明显下降(表 13, Entries 11, 12). 当芳环的间位带有供 电子基团 $\left(\mathrm{CH}_{3}\right)$ 或吸电子基 $\left(\mathrm{Cl} 、 \mathrm{NO}_{2}\right)$ 时，分别以 $90 \%$ 、 93\%、96\%的收率并大于 $99: 1$ 的非对映选择性得到目

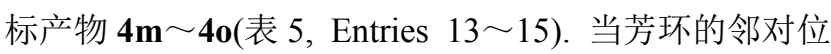
带有吸电子基团 $\mathrm{Cl}$ 时，反应以 $92 \%$ 的收率得到目标产 物 4p, 但反应的非对映选择性明显下降至 87：13(表 5, Entry 16). 其次, 考察 $\mathrm{R}$ 为杂环时对反应产物的收率及 非对映选择性的影响. 当 $\mathrm{R}$ 为 1 -䒺基、2-噻吩基时,

表 5 底物拓展 ${ }^{a}$

Table 5 Substrate scope in the reaction

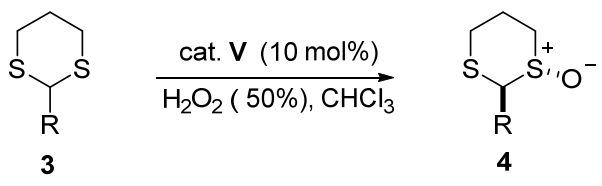

\begin{tabular}{clcccc}
\hline Entry & \multicolumn{1}{c}{$\mathrm{R}$} & Product & Time/h & Yield $^{b} \%$ & $\begin{array}{c}d . r . \\
(\text { trans }: \text { cis })^{c}\end{array}$ \\
\hline 1 & $\mathrm{C}_{6} \mathrm{H}_{5}$ & $\mathbf{4 a}$ & 1.0 & 95 & $>99: 1$ \\
2 & $p-\mathrm{FC}_{6} \mathrm{H}_{4}$ & $\mathbf{4 b}$ & 1.5 & 94 & $>99: 1$ \\
3 & $p-\mathrm{ClC}_{6} \mathrm{H}_{4}$ & $\mathbf{4 c}$ & 1.5 & 95 & $>99: 1$ \\
4 & $p-\mathrm{BrC}_{6} \mathrm{H}_{4}$ & $\mathbf{4 d}$ & 2.0 & 92 & $>99: 1$ \\
5 & $p-\mathrm{CF}_{3} \mathrm{C}_{6} \mathrm{H}_{4}$ & $\mathbf{4 e}$ & 2.0 & 97 & $>99: 1$ \\
6 & $p-\mathrm{CNC}_{6} \mathrm{H}_{4}$ & $\mathbf{4 f}$ & 2.5 & 92 & $>99: 1$ \\
7 & $p-\mathrm{NO}_{2} \mathrm{C}_{6} \mathrm{H}_{4}$ & $\mathbf{4 g}$ & 1.5 & 86 & $>99: 1$ \\
8 & $p-\mathrm{CH}_{3} \mathrm{C}_{6} \mathrm{H}_{4}$ & $\mathbf{4 h}$ & 2.5 & 93 & $>99: 1$ \\
9 & $p-i-\mathrm{PrC}_{6} \mathrm{H}_{4}$ & $\mathbf{4 i}$ & 2.5 & 96 & $>99: 1$ \\
10 & $o-\mathrm{OCH}_{3} \mathrm{C}_{6} \mathrm{H}_{4}$ & $\mathbf{4 j}$ & 2.5 & 94 & $94: 6$ \\
11 & $o-\mathrm{FC}_{6} \mathrm{H}_{4}$ & $\mathbf{4 k}$ & 3.5 & 81 & $92: 8$ \\
12 & $o-\mathrm{ClC}_{6} \mathrm{H}_{4}$ & $\mathbf{4 l}$ & 3.0 & 82 & $89: 11$ \\
13 & $m-\mathrm{CH}_{3} \mathrm{C}_{6} \mathrm{H}_{4}$ & $\mathbf{4 m}$ & 2.0 & 90 & $>99: 1$ \\
14 & $m-\mathrm{ClC}_{6} \mathrm{H}_{4}$ & $\mathbf{4 n}$ & 2.0 & 93 & $>99: 1$ \\
15 & $m-\mathrm{NO}_{2} \mathrm{C}_{6} \mathrm{H}_{4}$ & $\mathbf{4 o}$ & 2.5 & 96 & $>99: 1$ \\
16 & $2,4-\mathrm{Cl}_{2} \mathrm{C}_{6} \mathrm{H}_{3}$ & $\mathbf{4 p}$ & 2.0 & 92 & $87: 13$ \\
17 & $1-\mathrm{Naphthyl}_{10}$ & $\mathbf{4 q}$ & 2.0 & 93 & $>99: 1$ \\
18 & $2-\mathrm{Thienyl}$ & $\mathbf{4 r}$ & 2.0 & 93 & $>99: 1$ \\
\hline
\end{tabular}

${ }^{a}$ Reactions were performed with $3(0.5 \mathrm{mmol}), \mathrm{H}_{2} \mathrm{O}_{2}\left(50 \%\right.$ in $\mathrm{H}_{2} \mathrm{O}, 1.2$ equiv.) and catalyst $(10 \mathrm{~mol} \%)$ in solvent $(1.0 \mathrm{~mL}) .{ }^{b}$ Isolated yield. ${ }^{c}$ Determined by ${ }^{1} \mathrm{H}$ NMR 
均以 $92 \% \sim 93 \%$ 的收率及大于 $99: 1$ 的非对映选择性得 到目标化合物 4q 和 $4 \mathbf{r}$ (表 5, Entries 17, 18).

从机理上讲磷酸二苯酯的膦氧官能团和羟基官能 团分别与过氧化氢分子以氢键的方式, 形成七元环络合 物, 同时硫醚分子进攻过氧化氢分子中的氧原子, 形成 亚砜分子. 过氧化氢分子在磷酸二苯酯的作用下并最终 释放出一分子水, 完成一个催化循环(图 1).

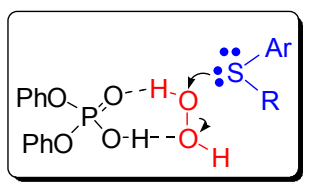

图 1 磷酸二苯酯催化硫醚氧化的过渡态模型

Figure 1 Transition state of diphenyl hydrogen phosphate catalyzed oxidation of thioether

\section{2 结论}

通过上述研究发现, 在室温条件下, 以过氧化氢水 溶液为氧源, 磷酸二苯酯能高效催化芳基烷基硫醚及 1,3-缩二硫醛的氧化反应, 能以良好到优秀的收率和高 立体选择性得到相应的亚砜类化合物. 通过条件的优 化, 基本上避免了过度氧化砜类副产物的生成. 所发展 的方法不仅产率高, 反应条件温和, 而且反应的区域选 择性和非对映选择性良好.

\section{3 实验部分}

\section{1 仪器与试剂}

核磁共振氢谱和碳谱采用 Varian Inova-400 或者 Varian ststem-300 型核磁共振仪, TMS 为内标, $\mathrm{CDCl}_{3}$ 为 溶剂; 质谱采用液质联动高分辨质谱仪 (Micromass GCT-TOF) 测定. 实验过程中所用的溶剂和试剂都是 AR 级, 柱层析采用 200 300 目的柱层析硅胶, 1,3-缩二 硫醛底物参考文献方法合成.

\section{2 合成亚砜的一般步骤}

将苯基烷基硫醚 $\mathbf{1 a} \sim \mathbf{1 h}$ 或 1,3-缩二硫醛 $\mathbf{3 a} \sim \mathbf{3 h}$ $(0.5 \mathrm{mmol})$, 磷酸二苯酯 $\mathbf{V}(12.5 \mathrm{mg}, 0.05 \mathrm{mmol})$ 溶于 1,2 -二氯乙烷 $(1 \mathrm{~mL})$ 或氯仿 $(1 \mathrm{~mL})$ 中, 搅拌 $2 \mathrm{~min}$ 之后加 入 $50 \%$ 的过氧化氢水溶液 $(57 \mu \mathrm{L}, 2.0$ equiv.)继续搅拌. $\mathrm{TLC}$ 检测反应结束后, 用 $\mathrm{NaHSO}_{3}$ 饱和水溶液淬灭反应. 将水 $(10 \mathrm{~mL})$ 加入到反应管中, 用乙酸乙酯萃取 (10 $\mathrm{mL} \times 3)$, 合并有机相, 无水 $\mathrm{Na}_{2} \mathrm{SO}_{4}$ 干燥. 浓缩后用制备 型 TLC [展开剂: $V$ (二氯甲烷) $: V$ (甲醇 $)=10 ： 1$ ], 得到 相应的亚砜化合物 $\mathbf{2 a} \sim \mathbf{2 h}$ 或 $\mathbf{4 a} \sim \mathbf{4 r}$.

苯基甲基亚砜(2a): 液体，产率 99\%. ${ }^{1} \mathrm{H}$ NMR (400 $\left.\mathrm{MHz}, \mathrm{CDCl}_{3}\right) \delta: 7.58$ (d, $\left.J=8.6 \mathrm{~Hz}, 2 \mathrm{H}\right), 7.36$ (t, $J=8.6$ $\mathrm{Hz}, 2 \mathrm{H}), 2.65$ (s, 3H); ${ }^{13} \mathrm{C}$ NMR $\left(100 \mathrm{MHz}, \mathrm{CDCl}_{3}\right) \delta$ :
145.6, 131.1, 129.4, 123.6, 44.0; ESI-MS $m / z: 141.0$ [M+ $\mathrm{H}^{+}$; HRMS-ESI calcd for $\mathrm{C}_{7} \mathrm{H}_{9} \mathrm{OS}[\mathrm{M}+\mathrm{H}]^{+}: 141.0374$, found 141.0370 .

4-甲氧基苯基甲基亚砜 (2b): 液体, 产率 $96 \% .{ }^{1} \mathrm{H}$ NMR (400 MHz, $\mathrm{CDCl}_{3}$ ) $\delta: 7.57$ (d, $\left.J=8.6 \mathrm{~Hz}, 2 \mathrm{H}\right), 7.00$ (d, $J=8.6 \mathrm{~Hz}, 2 \mathrm{H}), 3.83$ (s, 3H), 2.67 (s, 3H); ${ }^{13} \mathrm{C}$ NMR $\left(100 \mathrm{MHz}, \mathrm{CDCl}_{3}\right) \delta: 161.8,136.4,125.3,114.7,55.4$, 43.9; ESI-MS $m / z$ : $171.0[\mathrm{M}+\mathrm{H}]^{+}$; HRMS-ESI calcd for $\mathrm{C}_{8} \mathrm{H}_{11} \mathrm{O}_{2} \mathrm{~S}[\mathrm{M}+\mathrm{H}]^{+}$171.0480, found 171.0483,.

苯基苄基亚砜 (2c)：白色固体，产率 94\%. m.p. $114 \sim 116{ }^{\circ} \mathrm{C} ;{ }^{1} \mathrm{H}$ NMR $\left(400 \mathrm{MHz}, \mathrm{CDCl}_{3}\right) \delta: 7.46 \sim 7.36$ (m, $5 \mathrm{H}), 7.28 \sim 7.26(\mathrm{~m}, 3 \mathrm{H}), 7.24 \sim 6.97(\mathrm{~m}, 2 \mathrm{H}), 4.08 \sim$ $4.00(\mathrm{AB}, J=12.6 \mathrm{~Hz}, 2 \mathrm{H}) ;{ }^{13} \mathrm{C}$ NMR $\left(100 \mathrm{MHz}, \mathrm{CDCl}_{3}\right)$ $\delta: 142.6,131.1,130.7,130.3,129.0,128.7,128.5,128.3$, 128.1, 124.3, 63.4; ESI-MS $m / z: 216.9[\mathrm{M}+\mathrm{H}]^{+}$; HRMS-ESI calcd for $\mathrm{C}_{13} \mathrm{H}_{13} \mathrm{OS}[\mathrm{M}+\mathrm{H}]^{+}$217.0687, found 217.0688 .

4-溴苯基甲基亚砜(2d): 白色固体, 产率 93\%. m.p. 59 60 ${ }^{\circ} \mathrm{C} ;{ }^{1} \mathrm{H}$ NMR $\left(400 \mathrm{MHz}, \mathrm{CDCl}_{3}\right) \delta: 7.67$ (d, $J=8.0$ $\mathrm{Hz}, 2 \mathrm{H}), 7.53(\mathrm{~d}, J=8.4 \mathrm{~Hz}, 2 \mathrm{H}), 2.72(\mathrm{~s}, 3 \mathrm{H}) ;{ }^{13} \mathrm{C}$ NMR $\left(100 \mathrm{MHz}, \mathrm{CDCl}_{3}\right) \delta: 144.6,132.3,125.2,124.9,43.86$, 43.83; ESI-MS $m / z(\%): 218.8$ (98) and 220.8 (100) [M+ $\mathrm{H}]^{+}$for ${ }^{79} \mathrm{Br}$ 和 ${ }^{81} \mathrm{Br}$ isotopic pattern; HRMS-ESI calcd for $\mathrm{C}_{7} \mathrm{H}_{8}{ }^{79} \mathrm{BrO}^{32} \mathrm{~S}[\mathrm{M}+\mathrm{H}]^{+} 218.9474$ found 218.9474 (98); HRMS-ESI calcd for $\mathrm{C}_{7} \mathrm{H}_{8}{ }^{81} \mathrm{BrO}^{34} \mathrm{~S}[\mathrm{M}+\mathrm{H}]^{+}$220.9459, found 220.9453 (100).

4-氯苯基甲基亚砜(2e)：液体，产率 $92 \% .{ }^{1} \mathrm{H}$ NMR $\left(400 \mathrm{MHz}, \mathrm{CDCl}_{3}\right) \delta: 7.59$ (d, $\left.J=8.4 \mathrm{~Hz}, 2 \mathrm{H}\right), 7.50$ (d, $J=$ $8.4 \mathrm{~Hz}, 2 \mathrm{H}), 2.72(\mathrm{~s}, 3 \mathrm{H}) ;{ }^{13} \mathrm{C}$ NMR $\left(100 \mathrm{MHz}, \mathrm{CDCl}_{3}\right) \delta$ : 144.0, 137.1, 129.5, 124.8, 43.9; ESI-MS $m / z$ (\%): 174.9 (100) and $176.9(38)[\mathrm{M}+\mathrm{H}]+$ for ${ }^{35} \mathrm{Cl}$ and ${ }^{37} \mathrm{Cl}$ isotopic pattern; HRMS-ESI calcd for $\mathrm{C}_{7} \mathrm{H}_{8}{ }^{35} \mathrm{ClO}^{32} \mathrm{~S}[\mathrm{M}+\mathrm{H}]^{+}$ 174.9979, found 174.9977 (100); HRMS-ESI calcd for $\mathrm{C}_{7} \mathrm{H}_{8}{ }^{37} \mathrm{ClO}^{34} \mathrm{~S}[\mathrm{M}+\mathrm{H}]^{+} \quad 176.9950$, found 176.9947 (38).

2-澳苯基甲基亚砜(2f): 液体, 产率 $99 \% .{ }^{1} \mathrm{H}$ NMR $\left(400 \mathrm{MHz}, \mathrm{CDCl}_{3}\right) \delta: 7.91(\mathrm{~d}, J=8.0 \mathrm{~Hz}, 1 \mathrm{H}), 7.56(\mathrm{~d}, J=$ $8.4 \mathrm{~Hz}, 2 \mathrm{H}), 7.54 \sim 7.35(\mathrm{~m}, 1 \mathrm{H}), 2.79(\mathrm{~s}, 3 \mathrm{H}) ;{ }^{13} \mathrm{C} \mathrm{NMR}$ $\left(100 \mathrm{MHz}, \mathrm{CDCl}_{3}\right) \delta: 145.1,132.8,132.2,128.6,125.5$, 118.3,114.4, 41.8; ESI-MS $m / z$ (\%): 218.8 (98) and 220.8 (100) $[\mathrm{M}+\mathrm{H}]^{+}$for ${ }^{79} \mathrm{Br}$ and ${ }^{81} \mathrm{Br}$ isotopic pattern; HRMS-ESI calcd for $\mathrm{C}_{7} \mathrm{H}_{8}{ }^{79} \mathrm{BrO}^{32} \mathrm{~S}[\mathrm{M}+\mathrm{H}]^{+}: 218.9474$, found 218.9472 (98); HRMS-ESI calcd for $\mathrm{C}_{7} \mathrm{H}_{8}{ }^{81} \mathrm{BrO}^{34} \mathrm{~S}$ $[\mathrm{M}+\mathrm{H}]^{+}$220.9459, found $220.9450(100)$.

4-甲基苯基甲基亚砜(2g): 白色固体，产率 90\%. m.p. $72 \sim 73{ }^{\circ} \mathrm{C} ;{ }^{1} \mathrm{H}$ NMR $\left(400 \mathrm{MHz}, \mathrm{CDCl}_{3}\right) \delta: 7.50$ (d, 
$J=8.0 \mathrm{~Hz}, 2 \mathrm{H}), 7.29$ (d, $J=8.4 \mathrm{~Hz}, 2 \mathrm{H}), 2.67$ (s, 3H), 2.38 (s, 3H); ${ }^{13} \mathrm{C}$ NMR (100 MHz, $\left.\mathrm{CDCl}_{3}\right) \delta: 142.3,141.4$, 129.9, 123.4, 43.9, 21.3; ESI-MS $m / z: 155.0[\mathrm{M}+\mathrm{H}]^{+}$; HRMS-ESI calcd for $\mathrm{C}_{8} \mathrm{H}_{11} \mathrm{OS}[\mathrm{M}+\mathrm{H}]^{+} 155.0531$, found 155.0527 .

苯基乙基亚砜(2h)：液体，产率 90\%. ${ }^{1} \mathrm{H}$ NMR (400 $\left.\mathrm{MHz}, \mathrm{CDCl}_{3}\right) \delta: 7.55 \sim 7.54(\mathrm{~m}, 2 \mathrm{H}), 7.47 \sim 7.44(\mathrm{~m}, 3 \mathrm{H})$, $2.87 \sim 2.74(\mathrm{~m}, 1 \mathrm{H}), 2.73 \sim 2.68(\mathrm{~m}, 1 \mathrm{H}), 1.15 \sim 1.11(\mathrm{~m}$, $3 \mathrm{H}) ;{ }^{13} \mathrm{C} \mathrm{NMR}\left(100 \mathrm{MHz}, \mathrm{CDCl}_{3}\right) \delta: 143.0,130.8,129.0$, 124.0, 50.1, 5.7; ESI-MS $m / z: 155.0[\mathrm{M}+\mathrm{H}]^{+}$; HRMS-ESI calcd for $\mathrm{C}_{8} \mathrm{H}_{11} \mathrm{OS}[\mathrm{M}+\mathrm{H}]^{+}$155.0531, found 155.0529.

反-2-苯基-1,3-二噻烷-1-氧化物(4a)：白色固体, 产 率 95\%, >20:1 dr. m.p. $136 \sim 137{ }^{\circ} \mathrm{C} ;{ }^{1} \mathrm{H}$ NMR (300 $\left.\mathrm{MHz}, \mathrm{CDCl}_{3}\right) \delta: 7.39 \sim 7.32(\mathrm{~m}, 5 \mathrm{H}), 4.54(\mathrm{~s}, 1 \mathrm{H}), 3.53-$ $3.49(\mathrm{~m}, 1 \mathrm{H}), 2.86 \sim 2.75(\mathrm{~m}, 1 \mathrm{H}), 2.72 \sim 2.58(\mathrm{~m}, 2 \mathrm{H})$, $2.47 \sim 2.41(\mathrm{~m}, 1 \mathrm{H}), 2.37 \sim 2.22(\mathrm{~m}, 1 \mathrm{H}) ;{ }^{13} \mathrm{C}$ NMR $(75$ $\left.\mathrm{MHz}, \mathrm{CDCl}_{3}\right) \delta$ : 133.6, 129.5, 129.3, 128.9, 69.8, 55.0, 31.6, 29.7; HRMS (ESI) calcd for $\mathrm{C}_{10} \mathrm{H}_{13} \mathrm{OS}_{2}[\mathrm{M}+\mathrm{H}]^{+}$ 213.0408, found 213.0400 .

反-2-(4'-氟苯基)-1,3-二噻烷-1-氧化物(4b): 白色固 体, 产率 94\%, >20:1 dr. m.p. 126 127 ${ }^{\circ} \mathrm{C} ;{ }^{1} \mathrm{H}$ NMR $\left(300 \mathrm{MHz}, \mathrm{CDCl}_{3}\right) \delta: 7.42 \sim 7.38(\mathrm{~m}, 2 \mathrm{H}), 7.12 \sim 7.06(\mathrm{~m}$, $2 \mathrm{H}), 4.54(\mathrm{~s}, 1 \mathrm{H}), 3.56(\mathrm{~d}, J=18.2 \mathrm{~Hz}, 1 \mathrm{H}), 2.88 \sim 2.65$ (m, 3H), 2.51 2.38 (m, 2H); ${ }^{13} \mathrm{C}$ NMR $\left(75 \mathrm{MHz}, \mathrm{CDCl}_{3}\right)$ $\delta: 165.1,161.8,130.8,116.6,116.3,69.0,54.9,31.7,29.7$; HRMS (ESI) calcd for $\mathrm{C}_{10} \mathrm{H}_{12} \mathrm{FOS}_{2}[\mathrm{M}+\mathrm{H}]^{+} 231.0314$, found 231.0304.

反-2-(4'-氯苯基)-1,3-二噻烷-1-氧化物(4c): 白色固 体, 产率 95\%, >20: $1 d r$. m.p. $144 \sim 146{ }^{\circ} \mathrm{C} ;{ }^{1} \mathrm{H}$ NMR $\left(300 \mathrm{MHz}, \mathrm{CDCl}_{3}\right) \delta: 7.37(\mathrm{~s}, 4 \mathrm{H}), 4.54(\mathrm{~s}, 1 \mathrm{H}), 3.58(\mathrm{~d}$, $J=16.8 \mathrm{~Hz}, 1 \mathrm{H}), 2.89 \sim 2.84(\mathrm{~m}, 1 \mathrm{H}), 2.80 \sim 2.76(\mathrm{~m}, 2 \mathrm{H})$, $2.72 \sim 2.67(\mathrm{~m}, 1 \mathrm{H}), 2.67 \sim 2.39(\mathrm{~m}, 1 \mathrm{H}) ;{ }^{13} \mathrm{C}$ NMR $(75$ $\left.\mathrm{MHz}, \mathrm{CDCl}_{3}\right) \delta: 135.6,132.1,130.3,129.6,69.1,55.0$, 31.6, 29.7; HRMS (ESI) calcd for $\mathrm{C}_{10} \mathrm{H}_{12} \mathrm{ClOS}_{2}[\mathrm{M}+\mathrm{H}]^{+}$ 247.0018, found 247.0010 .

反-2-(4'-溴苯基)-1,3-二噻烷-1-氧化物(4d)：白色固 体, 产率 92\%, >20:1 dr. m.p. $163 \sim 165{ }^{\circ} \mathrm{C} ;{ }^{1} \mathrm{H}$ NMR $\left(300 \mathrm{MHz}, \mathrm{CDCl}_{3}\right) \delta: 7.53(\mathrm{~d}, J=7.2 \mathrm{~Hz}, 2 \mathrm{H}), 7.29$ (d, $J=$ $1 \mathrm{~Hz}, 2 \mathrm{H}), 4.52$ (s, 1H), 3.55 (d, $J=17.2 \mathrm{~Hz}, 1 \mathrm{H}), 2.91-$ $2.82(\mathrm{~m}, 1 \mathrm{H}), 2.81 \sim 2.64(\mathrm{~m}, 1 \mathrm{H}), 2.53 \sim 2.48(\mathrm{~m}, 1 \mathrm{H})$, $2.40 \sim 2.32(\mathrm{~m}, 1 \mathrm{H}) ;{ }^{13} \mathrm{C}$ NMR $\left(75 \mathrm{MHz}, \mathrm{CDCl}_{3}\right) \delta: 132.6$, 132.5, 130.6, 123.8, 69.1, 54.9, 31.6, 29.7; HRMS (ESI) calcd for $\mathrm{C}_{10} \mathrm{H}_{12} \mathrm{BrOS}_{2}[\mathrm{M}+\mathrm{H}]^{+}$290.9513, 292.468, found 290.9510, 292.488 .

反-2-(三氟甲基苯基)-1,3-二噻烷-1-氧化物(4e): 白
色固体, 产率 97\%, $>20 ： 1 d r$. m.p. 99 $100{ }^{\circ} \mathrm{C} ;{ }^{1} \mathrm{H}$ NMR (300 MHz, $\left.\mathrm{CDCl}_{3}\right) \delta: 7.67(\mathrm{~d}, J=8.1 \mathrm{~Hz}, 2 \mathrm{H}), 7.55$ (d, $J=8.1 \mathrm{~Hz}, 2 \mathrm{H}), 4.62(\mathrm{~s}, 1 \mathrm{H}), 3.59$ (d, $J=12.9 \mathrm{~Hz}, 1 \mathrm{H})$, $2.90 \sim 2.68(\mathrm{~m}, 3 \mathrm{H}), 2.52 \sim 2.40(\mathrm{~m}, 2 \mathrm{H}) ;{ }^{13} \mathrm{C}$ NMR $(75$ $\left.\mathrm{MHz}, \mathrm{CDCl}_{3}\right) \delta: 137.6,131.8,131.3$ 129.5, 126.3, 122.2, 69.3, 55.0, 31.5, 29.7; HRMS (ESI) calcd for $\mathrm{C}_{11} \mathrm{H}_{12} \mathrm{~F}_{3} \mathrm{OS}_{2}$ $[\mathrm{M}+\mathrm{H}]^{+}$281.0282, found 281.0279;

反-2-(4'-氧基苯基)-1,3-二噻烷-1-氧化物(4f)：白色 固体, 产率 93\%, >20:1 dr. m.p. $137 \sim 138{ }^{\circ} \mathrm{C} ;{ }^{1} \mathrm{H}$ NMR (300 MHz, $\left.\mathrm{CDCl}_{3}\right) \delta: 7.70(\mathrm{~d}, J=8.4 \mathrm{~Hz}, 2 \mathrm{H}), 7.54$ (d, $J=8.4 \mathrm{~Hz}, 2 \mathrm{H}), 4.62(\mathrm{~s}, 1 \mathrm{H}), 3.60(\mathrm{~d}, J=12.9 \mathrm{~Hz}, 1 \mathrm{H})$, $2.96 \sim 2.70(\mathrm{~m}, 3 \mathrm{H}), 2.58 \sim 2.36(\mathrm{~m}, 2 \mathrm{H}) ;{ }^{13} \mathrm{C}$ NMR $(75$ $\left.\mathrm{MHz}, \mathrm{CDCl}_{3}\right) \delta: 138.9,133.0,129.8,118.4,113.3,69.3$, 55.0, 31.4, 29.7; HRMS (ESI) calcd for $\mathrm{C}_{11} \mathrm{H}_{12} \mathrm{NOS}_{2}[\mathrm{M}+$ $\mathrm{H}]^{+}$238.0360, found 238.0345 .

反-2-(4'-硝基苯基)-1,3-二噻烷-1-氧化物(4g)：白色 固体, 产率 86\%, >20:1 dr. m.p. 162 163 ${ }^{\circ} \mathrm{C} ;{ }^{1} \mathrm{H}$ NMR $\left(300 \mathrm{MHz}, \mathrm{CDCl}_{3}\right) \delta: 8.26(\mathrm{~d}, J=8.4 \mathrm{~Hz}, 2 \mathrm{H}), 7.60$ (d, $J=8.7 \mathrm{~Hz}, 2 \mathrm{H}), 4.68(\mathrm{~s}, 1 \mathrm{H}), 3.62(\mathrm{~d}, J=12.6 \mathrm{~Hz}, 1 \mathrm{H})$, $2.94 \sim 2.72(\mathrm{~m}, 3 \mathrm{H}), 2.55 \sim 2.39(\mathrm{~m}, 2 \mathrm{H}) ;{ }^{13} \mathrm{C}$ NMR $(75$ $\left.\mathrm{MHz}, \mathrm{CDCl}_{3}\right) \delta: 148.5,140.8,130.1,124.4,69.0,55.1$, 31.5, 29.7; HRMS (ESI) calcd for $\mathrm{C}_{10} \mathrm{H}_{12} \mathrm{NO}_{3} \mathrm{~S}_{2}[\mathrm{M}+\mathrm{H}]^{+}$ 258.0259 , found 258.0253 .

反-2-(4'-甲基苯基)-1,3-二噻烷-1-氧化物(4h): 白色 固体, 产率 93\%, >20:1 dr. m.p. $165 \sim 167{ }^{\circ} \mathrm{C} ;{ }^{1} \mathrm{H}$ NMR (300 MHz, $\left.\mathrm{CDCl}_{3}\right) \delta: 7.31(\mathrm{~d}, J=8.1 \mathrm{~Hz}, 2 \mathrm{H}), 7.20$ (d, $J=8.1 \mathrm{~Hz}, 2 \mathrm{H}), 4.52(\mathrm{~s}, 1 \mathrm{H}), 3.54(\mathrm{~d}, J=12.9 \mathrm{~Hz}, 1 \mathrm{H})$, $2.91 \sim 2.77(\mathrm{~m}, 1 \mathrm{H}), 2.74 \sim 2.63(\mathrm{~m}, 2 \mathrm{H}) 2.53 \sim 2.40(\mathrm{~m}$, $1 \mathrm{H}), 2.39$ (s, 3H); ${ }^{13} \mathrm{C} \mathrm{NMR}\left(75 \mathrm{MHz}, \mathrm{CDCl}_{3}\right) \delta: 139.6$, 139.3, 130.5, 130.1, 128.8, 69.7, 54.9, 31.7, 29.8, 21.5; HRMS (ESI) calcd for $\mathrm{C}_{11} \mathrm{H}_{15} \mathrm{OS}_{2}[\mathrm{M}+\mathrm{H}]^{+} 227.0564$, found 227.0561 .

反-2-(4'-异丙苯基)-1,3-二噻烷-1-氧化物(4i): 白色 固体, 产率 96\%, >20:1 dr. m.p. $105 \sim 107{ }^{\circ} \mathrm{C} ;{ }^{1} \mathrm{H}$ NMR (400 MHz, $\left.\mathrm{CDCl}_{3}\right) \delta: 7.32(\mathrm{~d}, J=8.4 \mathrm{~Hz}, 2 \mathrm{H}), 7.23$ (d, $J=8.0 \mathrm{~Hz}, 2 \mathrm{H}), 4.53(\mathrm{~s}, 1 \mathrm{H}), 3.55 \sim 3.51(\mathrm{~m}, 1 \mathrm{H}), 2.87$ (s, $1 \mathrm{H}), 2.85(\mathrm{~s}, 1 \mathrm{H}), 2.81 \sim 2.76(\mathrm{~m}, 1 \mathrm{H}), 2.69(\mathrm{~m}, 1 \mathrm{H})$, $2.6(\mathrm{~m}, 1 \mathrm{H}), 2.65 \sim 2.44(\mathrm{~m}, 1 \mathrm{H}), 1.22(\mathrm{~d}, J=7.2 \mathrm{~Hz}, 6 \mathrm{H})$; ${ }^{13} \mathrm{C}$ NMR (100 MHz, $\left.\mathrm{CDCl}_{3}\right) \delta: 149.8,130.3,128.4,127.0$, 69.2, 54.5, 33.7, 31.2, 29.4, 23.6; HRMS (ESI) calcd for $\mathrm{C}_{13} \mathrm{H}_{19} \mathrm{OS}_{2}[\mathrm{M}+\mathrm{H}]^{+}$255.0877, found 255.0874.

反-2-(2'-甲氧基苯基)-1,3-二噻烷-1-氧化物 (4j): 白 色固体, 产率 94\%, 16:1 dr. m.p. $149 \sim 151{ }^{\circ} \mathrm{C} ;{ }^{1} \mathrm{H}$ NMR (300 MHz, $\left.\mathrm{CDCl}_{3}\right) \delta: 7.41(\mathrm{~d}, J=7.8 \mathrm{~Hz}, 1 \mathrm{H})$, $7.34 \sim 7.29(\mathrm{~m}, 1 \mathrm{H}), 7.01(\mathrm{t}, J=7.5 \mathrm{~Hz}, 1 \mathrm{H}), 6.92(\mathrm{~d}, J=$ 
$8.4 \mathrm{~Hz}, 1 \mathrm{H}), 5.28(\mathrm{~s}, 1 \mathrm{H}), 3.87(\mathrm{~s}, 3 \mathrm{H}), 3.58 \sim 3.54(\mathrm{~m}, 1 \mathrm{H})$, $2.96 \sim 2.76(\mathrm{~m}, 2 \mathrm{H}), 2.66 \sim 2.50(\mathrm{~m}, 2 \mathrm{H}), 2.38 \sim 2.34(\mathrm{~m}$, $1 \mathrm{H}) ;{ }^{13} \mathrm{C}$ NMR (75 MHz, $\left.\mathrm{CDCl}_{3}\right) \delta: 157.9,130.5,129.1$, 122.0, 121.5, 111.5, 62.0, 56.1, 55.0, 31.9, 30.0; HRMS (ESI) calcd for $\mathrm{C}_{11} \mathrm{H}_{15} \mathrm{O}_{2} \mathrm{~S}_{2}[\mathrm{M}+\mathrm{H}]^{+}$243.0513, found 243.0509 .

反-2-(2'-氟苯基)-1,3-二噻烷-1-氧化物(4k): 白色固 体, 产率 81\%, $12: 1 d r$. m.p. $117 \sim 118{ }^{\circ} \mathrm{C} ;{ }^{1} \mathrm{H}$ NMR $\left(400 \mathrm{MHz}, \mathrm{CDCl}_{3}\right) \delta: 7.45(\mathrm{t}, J=7.2 \mathrm{~Hz}, 1 \mathrm{H}), 7.36 \sim 7.31$ $(\mathrm{m}, 1 \mathrm{H}), 7.22 \sim 7.19(\mathrm{~m}, 1 \mathrm{H}), 7.14 \sim 7.09(\mathrm{~m}, 1 \mathrm{H}), 5.02(\mathrm{~s}$, $1 \mathrm{H}), 3.59 \sim 3.56(\mathrm{~m}, 1 \mathrm{H}), 2.94 \sim 2.79(\mathrm{~m}, 1 \mathrm{H}), 2.78-2.64$ (m, $1 \mathrm{H}), 2.54 \sim 2.50(\mathrm{~m}, 1 \mathrm{H}), 2.38 \sim 2.34(\mathrm{~m}, 1 \mathrm{H}) ;{ }^{13} \mathrm{C}$ NMR $\left(75 \mathrm{MHz}, \mathrm{CDCl}_{3}\right) \delta$ : 162.8, 159.5, 131.1, 129.4, 125.3, 125.2, 121.3, 121.1, 116.4, 116.1, 61.9, 55.1, 31.7, 29.9; HRMS (ESI) calcd. for $\left(\mathrm{C}_{10} \mathrm{H}_{12} \mathrm{FOS}_{2}\right)[\mathrm{M}+\mathrm{H}]^{+}$ 231.0314, found 231.0314

反-2-(2'-氯苯基)-1,3-二噻烷-1-氧化物(4I): 白色固 体, 产率 94\%, $8.7: 1 d r$. m.p. $134 \sim 136{ }^{\circ} \mathrm{C} ;{ }^{1} \mathrm{H}$ NMR $\left(300 \mathrm{MHz}, \mathrm{CDCl}_{3}\right) \delta: 7.51(\mathrm{~d}, J=7.2 \mathrm{~Hz}, 1 \mathrm{H}), 7.43$ (d, $J=$ $7.5 \mathrm{~Hz}, 1 \mathrm{H}), 7.35 \sim 7.25(\mathrm{~m}, 2 \mathrm{H}), 5.27(\mathrm{~s}, 1 \mathrm{H}), 3.61 \sim 3.57$ $(\mathrm{m}, 1 \mathrm{H}), 2.98 \sim 2.81(\mathrm{~m}, 2 \mathrm{H}), 2.67 \sim 2.63(\mathrm{~m}, 1 \mathrm{H}), 2.55 \sim$ $2.50(\mathrm{~m}, 1 \mathrm{H}), 2.39 \sim 2.34(\mathrm{~m}, 1 \mathrm{H}) ;{ }^{13} \mathrm{C}$ NMR $(75 \mathrm{MHz}$, $\left.\mathrm{CDCl}_{3}\right) \delta: 135.2,131.7,130.4,129.5128 .1,65.4,55.1$, 31.9, 30.0; HRMS (ESI) calcd for $\mathrm{C}_{10} \mathrm{H}_{12} \mathrm{ClOS}_{2}[\mathrm{M}+\mathrm{H}]^{+}$ 247.0018, found 247.0013 .

反-2-(3'-甲基苯基)-1,3-二噻烷-1-氧化物(4m): 白色 固体, 产率 90\%, > 20:1 dr. m.p. 143 $144{ }^{\circ} \mathrm{C} ;{ }^{1} \mathrm{H}$ NMR $\left(300 \mathrm{MHz}, \mathrm{CDCl}_{3}\right) \delta: 7.31 \sim 7.16(\mathrm{~m}, 4 \mathrm{H}), 4.52(\mathrm{~s}$, $1 \mathrm{H}), 3.55(\mathrm{~d}, J=12.9 \mathrm{~Hz}, 1 \mathrm{H}), 2.86 \sim 2.81(\mathrm{~m}, 1 \mathrm{H}), 2.79 \sim$ $2.63(\mathrm{~m}, 2 \mathrm{H}), 2.53 \sim 2.47(\mathrm{~m}, 2 \mathrm{H}), 2.41 \sim 2.15(\mathrm{~m}, 4 \mathrm{H})$; ${ }^{13} \mathrm{C}$ NMR $\left(75 \mathrm{MHz}, \mathrm{CDCl}_{3}\right) \delta: 139.1,133.4,130.4,129.5$, 129.2, 126.0, 69.9, 55.0, 31.7, 31.1, 29.8, 21.6; HRMS (ESI) calcd for $\mathrm{C}_{11} \mathrm{H}_{15} \mathrm{OS}_{2}[\mathrm{M}+\mathrm{H}]^{+}$227.0564, found 227.0560 .

反-2-(3'-氯苯基)-1,3-二噻烷-1-氧化物(4n): 白色固 体, 产率 93\%, >20: $1 d r$. m.p. $118 \sim 119{ }^{\circ} \mathrm{C} ;{ }^{1} \mathrm{H}$ NMR $\left(300 \mathrm{MHz}, \mathrm{CDCl}_{3}\right) \delta: 7.42(\mathrm{~s}, 1 \mathrm{H}) 7.34 \sim 7.31(\mathrm{~m}, 3 \mathrm{H})$, $4.52(\mathrm{~s}, 1 \mathrm{H}), 3.57(\mathrm{~d}, J=12.6 \mathrm{~Hz}, 1 \mathrm{H}), 2.91 \sim 2.66(\mathrm{~m}$, $3 \mathrm{H}), 2.54 \sim 2.33(\mathrm{~m}, 2 \mathrm{H}) ;{ }^{13} \mathrm{C} \mathrm{NMR}\left(75 \mathrm{MHz}, \mathrm{CDCl}_{3}\right) \delta$ : 135.5, 135.2, 130.6, 129.8, 129.1, 127.3, 69.2, 55.0, 31.6, 29.4; HRMS (ESI) calcd for $\mathrm{C}_{10} \mathrm{H}_{12} \mathrm{ClOS}_{2}[\mathrm{M}+\mathrm{H}]^{+}$ 247.0018, found 247.0017 .

反-2-(3'-硝基苯基)-1,3-二噻烷-1-氧化物(4o): 白色 固体, 产率 96\%, > $20: 1 d r$. m.p. $153 \sim 155{ }^{\circ} \mathrm{C} ;{ }^{1} \mathrm{H}$ NMR (300 MHz, $\left.\mathrm{CDCl}_{3}\right) \delta: 8.23(\mathrm{~d}, J=8.1 \mathrm{~Hz}, 2 \mathrm{H}), 7.75$ (d, $J=8.1 \mathrm{~Hz}, 2 \mathrm{H}), 7.58(\mathrm{t}, J=8.1 \mathrm{~Hz}, 1 \mathrm{H}), 4.67(\mathrm{~s}, 1 \mathrm{H})$, $3.60(\mathrm{~d}, J=12.9 \mathrm{~Hz}, 1 \mathrm{H}), 2.93 \sim 2.70(\mathrm{~m}, 3 \mathrm{H}), 2.58 \sim 2.53$ (m, 1H), 2.42 2.37 (m, 1H); ${ }^{13} \mathrm{C} \mathrm{NMR}\left(75 \mathrm{MHz}, \mathrm{CDCl}_{3}\right)$ $\delta: 148.7,135.8,135.2,130.4,124.5,124.1,68.8,55.0$, 31.5, 29.7; HRMS (ESI) calcd. for $\mathrm{C}_{10} \mathrm{H}_{12} \mathrm{NO}_{3} \mathrm{~S}_{2} \quad[\mathrm{M}+\mathrm{H}]$ 258.0259 , found 258.0254 .

反-2-(2',4'-二氯苯基)-1,3-二噻烷-1-氧化物(4p): 白 色固体, 产率 92\%, 6.6:1 dr. m.p. $177 \sim 179{ }^{\circ} \mathrm{C} ;{ }^{1} \mathrm{H}$ NMR $\left(300 \mathrm{MHz}, \mathrm{CDCl}_{3}\right) \delta: 7.45 \sim 7.42(\mathrm{~m}, 2 \mathrm{H}), 7.32 \sim$ 7.29 (m, 1H), $5.18(\mathrm{~s}, 1 \mathrm{H}), 3.58(\mathrm{~d}, J=12.6 \mathrm{~Hz}, 1 \mathrm{H})$, $2.97 \sim 2.80(\mathrm{~m}, 2 \mathrm{H}), 2.70 \sim 2.63(\mathrm{~m}, 1 \mathrm{H}), 2.54 \sim 2.497(\mathrm{~m}$, 1H), $2.493 \sim 2.32(\mathrm{~m}, 1 \mathrm{H}) ;{ }^{13} \mathrm{C}$ NMR $\left(75 \mathrm{MHz}, \mathrm{CDCl}_{3}\right) \delta$ : 135.9, 135.7, 130.5, 130.3, 130.2, 128.4, 64.8, 55.1, 31.8, 29.9; HRMS (ESI) calcd for $\mathrm{C}_{10} \mathrm{H}_{11} \mathrm{Cl}_{2} \mathrm{OS}_{2}[\mathrm{M}+\mathrm{H}]^{+}$ 280.9628 , found 280.9624.

反-2-(1'-䒺基)-1,3-二噻烷-1-氧化物(4q): 白色固体, 产率 93\%, > $20: 1 d r$. m.p. $182 \sim 183{ }^{\circ} \mathrm{C} ;{ }^{1} \mathrm{H}$ NMR $(400$ $\left.\mathrm{MHz}, \mathrm{CDCl}_{3}\right) \delta: 8.28(\mathrm{~d}, J=8.4 \mathrm{~Hz}, 1 \mathrm{H}), 7.88 \sim 7.85(\mathrm{~m}$, $2 \mathrm{H}), 7.71(\mathrm{~d}, J=6.8 \mathrm{~Hz}, 1 \mathrm{H}), 7.60 \sim 7.56(\mathrm{~m}, 1 \mathrm{H}), 7.53 \sim$ $7.48(\mathrm{~m}, 2 \mathrm{H}), 5.40(\mathrm{~s}, 1 \mathrm{H}), 3.63 \sim 3.60(\mathrm{~m}, 1 \mathrm{H}), 2.94 \sim$ $2.83(\mathrm{~m}, 2 \mathrm{H}), 2.65 \sim 2.62(\mathrm{~m}, 1 \mathrm{H}), 2.49 \sim 2.34(\mathrm{~m}, 2 \mathrm{H})$; ${ }^{13} \mathrm{C} \mathrm{NMR}\left(100 \mathrm{MHz}, \mathrm{CDCl}_{3}\right) \delta: 134.2,131.9,130.0,129.8$, 129.4, 129.1, 126.8, 126.3, 125.6, 123.4, 55.5, 32.1, 31.1, 29.9; HRMS (ESI) calcd for $\mathrm{C}_{14} \mathrm{H}_{15} \mathrm{OS}_{2}[\mathrm{M}+\mathrm{H}]^{+}$ 263.0564, found 263.0555 .

反-2-噻吩基-1,3-二噻烷-1-氧化物(4r): 白色固体, 产率 93\%, > $20: 1 d r$. m.p. $125 \sim 127{ }^{\circ} \mathrm{C} ;{ }^{1} \mathrm{H}$ NMR $(300$ $\left.\mathrm{MHz}, \mathrm{CDCl}_{3}\right) \delta: 7.34(\mathrm{~d}, J=5.1 \mathrm{~Hz}, 1 \mathrm{H}), 7.21(\mathrm{~d}, J=5.1$, $1 \mathrm{H}), 7.02(\mathrm{~d}, J=5.1,3.6 \mathrm{~Hz}, 1 \mathrm{H}), 4.83(\mathrm{~s}, 1 \mathrm{H}), 3.48(\mathrm{~d}$, $J=12.9 \mathrm{~Hz}, 1 \mathrm{H}), 2.86 \sim 2.81(\mathrm{~m}, 1 \mathrm{H}), 2.78 \sim 2.66(\mathrm{~m}, 2 \mathrm{H})$, $2.47 \sim 2.33(\mathrm{~m}, 1 \mathrm{H}), 2.28 \sim 2.14(\mathrm{~m}, 1 \mathrm{H}) ;{ }^{13} \mathrm{C}$ NMR $(75$ $\left.\mathrm{MHz}, \mathrm{CDCl}_{3}\right) \delta: 135.2,128.4,127.6,127.5,64.7,54.4$, 31.77, 28.8; HRMS (ESI) calcd for $\mathrm{C}_{8} \mathrm{H}_{11} \mathrm{OS}_{3}[\mathrm{M}+\mathrm{H}]^{+}$ 218.9972, found 218.9963 .

辅助材料(Supporting Information) 化合物 $\mathbf{2 a} \sim 2 \mathrm{~h}$ 和 4a $\sim \mathbf{4 r}$ 的谱图. 这些材料可以免费从本刊网站(http:// sioc-journal. cn/)上下载.

\section{References}

[1] (a) Legros, J.; Dehli, J. R.; Bolm, C. Adv. Synth. Catal. 2005, 347, 19.

(b) Fernandez, I.; Khiar, N. Chem. Rev. 2003, 103, 3651.

[2] (a) Holland, H. L. Chem. Rev. 1988, 88, 473.

(b) Block, E. Angew. Chem., Int. Ed. Engl. 1992, 31, 1135.

(c) Spencer, C. M.; Faulds, D. Drugs 2000, 103, 321.

[3] For selected examples see: 
(a) Di Furia, F.; Modena, G.; Seraglia, R. Synthesis 1984, 325.

(b) Pitchen, P.; Dunach, E.; Desmukh, M. N.; Kagan, H. B. J. Am. Chem. Soc. 1984, 106, 8188.

(c) Samuel, O.; Ronan, B.; Kagan, H. B. J. Organomet. Chem. 1989, 370, 43.

(d) Palucki, M.; Hanson, P.; Jacobsen, E. N. Tetrahedron Lett. 1992, 33, 7111 .

(e) Komatsu, N.; Hashizume, M.; Sugita, T.; Uemura, S. J. Org. Chem. 1993, 58, 4529.

(f) Bolm, C.; Bienewald, F. Angew. Chem., Int. Ed. Engl. 1995, 34, 2640.

(g) Kokubo, C.; Katsuki, T. Tetrahedron 1996, 52, 13895.

(h) Wang, X.; Wang, X.; Guo, H.; Wang, Z.; Ding, K. Chem. Eur. J. 2005, 11, 4078 .

(i) Mba, M.; Prins, L. J.; Licini, G. Org. Lett. 2007, 9, 21.

[4] Russo, A.; Lattanzia, A. Adv. Synth. Catal. 2009, 351, 521.

[5] Minidis, A. B. E.; Bäckvall, J.-E. Chem. Eur. J. 2001, 7, 297.

[6] Imada, Y.; Iida, H.; Ono, S. J. Am. Chem. Soc. 2003, 125, 2868.

[7] [7] Lindén, A. A.; Kr üger, L.; Bäckvall, J.-E. J. Org. Chem. 2003, $68,5890$.

[8] Lindén, A. A.; Johansson, M.; Hermanns, N. J. Org. Chem. 2006, 71,3849 .
[9] Kienle, M.; Argyrakis, W.; Baro, A.; Laschat, S. Tetrahedron Lett. 2008, 49, 1971.

[10] Selvam, J. J. P.; Suresh, V.; Rajesh, K.; Babu, D. C.; Suryakiran, N.; Venkateswarlu, Y. Tetrahedron Lett. 2008, 49, 3463.

[11] Carnell, A. J.; Clegg, W.; Johnstone, R. A. W.; Parsy, C. C.; Sanderson, W. R. Tetrahedron 2000, 56, 6571.

[12] Baumstark, A. L.; Chen, Y.-X.; Vasquez, P. C.; Yang, Y.-C. Heteroat. Chem. 1998, 9, 75.

[13] Shukla, V. G.; Salgaonkar, P. D.; Akamanchi. K. G. J. Org. Chem. 2003, 68, 5422.

[14] Qian, W.; Pei, L. Synlett 2006, 709.

[15] Tohma, H.; Takizawa, S.; Watanabe, H.; Kita, Y. Tetrahedron Lett. 1998, 39, 4547.

[16] Varma, R. S.; Saini, R. K.; Dahiya, R. J. Chem. Res., Synop. 1998, 120.

[17] Bulman Page, P. C.; Wilkes, R. D.; Namwindwa, E. S.; Witty, M. J. Tetrahedron 1996, 52, 2125.

[18] (a) Liao, S.; Čorić, I.; Wang, Q.; List, B. J. Am. Chem. Soc. 2012, 134, 10765

(b) Liu, Z.-M.; Zhao, H.; Li, M.-Q.; Lan Y.-B.; Yao; Q.-B.; Tao, J.-C.; Wang, X.-W. Adv. Synth. Catal. 2012, 354, 1012.

(Qin, X.) 